Abstracted/indexed in Academic Search Complete, Asia Journals Online, Bangladesh Journals Online, Biological Abstracts, BIOSIS Previews, CAB

Abstracts, Current Abstracts, Directory of Open Access Journals, EMBASE/Excerpta Medica, Google Scholar, HINARI (WHO), International

Pharmaceutical Abstracts, Open J-gate, Science Citation Index Expanded, SCOPUS and Social Sciences Citation Index;

ISSN: $1991-0088$

\title{
Antitumor and apoptotic effects of bergaptol are mediated via mitochondrial death pathway and cell cycle arrest in human breast carcinoma cells
}

\section{Zhi-Cheng Ge1, Xiang Qu'1, He-Fen Yu², Hui-Ming Zhang', Zi-Han Wang1 and Zhong-Tao Zhang 1}

\author{
${ }^{1}$ Department of General Surgery, Beijing Friendship Hospital, Capital University of Medical Science, \\ Beijing 100 050, China; ${ }^{2}$ Department of Biochemistry and Molecular Biology, School of Basic Medicine, \\ Capital Medical University; Cancer Institute of Capital Medical University; Beijing Key Laboratory for \\ Cancer Invasion and Metastasis Research. Beijing 100 069, China.
}

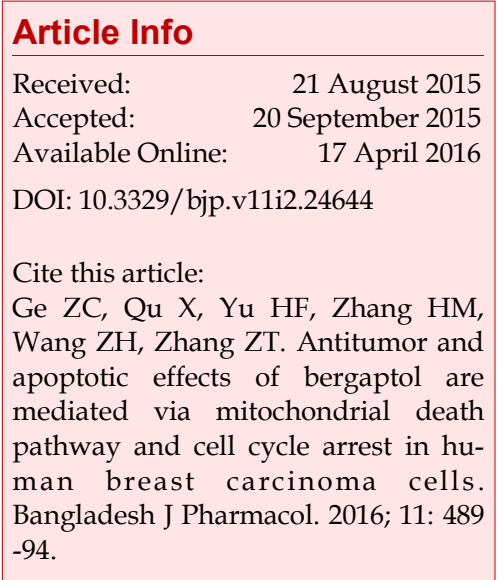

\section{Abstract}

The aim of the present research work was to investigate the anti-cancer and apoptotic effects of bergaptol in human breast cancer cells (MCF-7). The effects on cell cycle arrest and caspase activation were evaluated. MTT assay was used to evaluate the effect of the compound on cell viability. Cellular morphology was demonstrated by fluorescence microscopy. Flow cytometry was used to analyze effect of bergaptol on cell cycle and apoptosis. The results revealed that bergaptol induced dose-dependent cytotoxic effect on MCF-7 cell viability showing $\mathrm{IC}_{50}$ value of $52.2 \mu \mathrm{M}$. Bergaptol induced both early and late apoptosis in concentration-dependent manner. After treatment with bergaptol, an increase in the proportion of cells in the S-phase (37.2, 45.3 and $65.1 \%$ as compared to $28.6 \%$ in untreated cells) and a reduction in the fraction of cells in the G1 phase $(44.1,41.6$ and $35.2 \%$ as compared to $51.2 \%$ in the untreated cells) was observed.
\end{abstract}

\section{Introduction}

Breast cancer is responsible for around $15 \%$ of all cancer -related deaths in females rendering it one of the deadliest cancers (Jemal et al., 2011; Eccles et al., 2013). Treatment of breast cancer constitutes conventional treatment options including surgery, chemotherapy and radiotherapy among others. Chemotherapy has always been the most commonly used treatment regimen for breast cancer. However, most of the anti-cancer drugs kill normal cells along with cancerous cells and are nonselective (Conforti and Menichini, 2011). Additionally, multidrug resistance and quick removal of drugs from the systemic circulation results in reduced chemotherapeutic efficacy in tumor cells and hence further complicating the treatment process of cancer patients (Guzman-Villanueva et al., 2013). As such, there is an urgent need for chemotherapeutic agents with low toxicity and high selectivity for targeting malignant cells.

Natural products have always played a crucial role in anti-cancer drug discovery with about $65 \%$ of anticancer drugs derived from natural sources especially plant derived products. A number of Chinese medicinal plants have been reported to be effective against various cancers (Newman and Cragg, 2007).

The objective of the current research work was to investigate the anti-cancer effects of bergaptol in MCF-7 human breast cancer cells along with demonstrating the mode of action of these molecules by evaluating their effects on apoptosis, mitochondrial death pathway and cell cycle arrest. No such work has been reported on bergaptol (Figure 1) in the literature. Bergaptol (4- 
hydroxyfuro[3, 2-g]chromen-7-one) is a naturally occurring furanocoumarin found in lemon and bergamot essential oils (Stanley and Vannier, 1957).

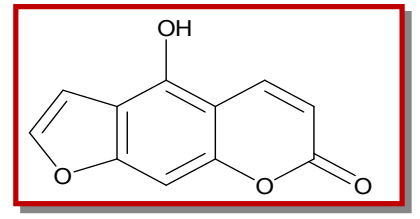

Figure 1: The chemical structure of bergaptol

\section{Materials and Methods}

\section{Materials}

Bergaptol was purchased from Sigma-Aldrich Inc. (USA). Dulbecco's modified Eagle's medium, fetal bovine serum, penicillin, streptomycin, trypsin, phosphate-buffered saline with calcium chloride, propidium iodide, DAPI and Tris base were obtained from Hangzhou Sijiqing Biological Engineering Materials Co., Ltd. (China). Annexin V-FITC-propidium iodide apoptosis detection kit was purchased from (Beyotime Institute of Biotechnology, China). All other chemicals and solvents used were of the highest purity grade. The cell-permeable pan-caspase inhibitor, the caspase-3 specific inhibitor, the caspase- 9 specific inhibitor, the caspase- 8 specific inhibitor, and the caspase- 2 specific inhibitor were purchased from (Santa Cruz Biotechnology, Inc., USA). The rabbit polyclonal antibodies (Abs) against caspase-3, caspase-8, the mouse monoclonal Abs (mAbs) against poly (ADP-ribose) polymerase (PARP), and the rabbit $\mathrm{mAb}$ against the active form of caspase-3 were purchased form Phar Mingen (Cell Signaling Technology, Inc.).

\section{Cell line}

The MCF-7 cell line was purchased from the American Type Culture Collection. These cells were cultured in Dulbecco's modified Eagle's medium containing 100 $\mathrm{mL} / \mathrm{L}$ fetal bovine serum with 100,000 U/L penicillin and $100 \mathrm{mg} / \mathrm{L}$ streptomycin under humidified atmosphere of $5 \% \mathrm{CO}_{2}$ at $37^{\circ} \mathrm{C}$. The medium was replaced every 24 hours and the cells were sub-cultured every 4 days.

\section{MTT assay for cell viability}

The effects of bergaptol on MCF-7 cell viability were studied by MTT assay. Cells $\left(2 \times 10^{6}\right.$ cells/well in 100 $\mu \mathrm{L}$ medium) were seeded into 96-well plates for 24 hours prior to drug treatment. After drug treatment with varying doses of bergaptol (0, 30, 60, 90 and 120 $\mu \mathrm{M})$ for 24 , the MTT solution was then added to the cell plates $(10 \mu \mathrm{L} ; 5 \mathrm{mg} / \mathrm{mL}$ in phosphate-buffered saline solution) for an additional 2 hours. DMSO $(200 \mu \mathrm{L})$ was used to solubilize the formazan crystals so formed and the absorbance was measured on a microplate reader (Bio-Rad, Hercules, CA) at a wavelength of $490 \mathrm{~nm}$.
Apoptosis and morphological study using fluorescence microscopy

MCF-7 ( 2 × $10^{6}$ cells $/ \mathrm{mL} /$ well) cells were treated with bergaptol at 30, 90 and $120 \mu \mathrm{M}$ concentration. After 48 hours incubation time, cells were centrifuged at 15,000 rpm for $20 \mathrm{~min}$. Resuspended pellet was dissolved in phosphate-buffered saline. The air dried smears were fixed in methanol at $-20^{\circ} \mathrm{C}$, stained with DAPI $(2 \mu \mathrm{g} /$ $\mathrm{mL}$ ) and kept at $37^{\circ} \mathrm{C}$ for $30 \mathrm{~min}$. Subsequent to phosphate-buffered saline wash, mounting was done with glycerol: phosphate-buffered saline (70:30) on cover slip and prepared slides were observed under fluorescence microscope (Olympus) using UV filter at 80x magnification. Taxol was used as a positive control.

\section{Annexin V-FITC/propidium iodide assay for quantita- tive estimation of cell apoptosis}

Apoptotic cells were quantified using an annexin Vfluorescein isothiocyanate (FITC)/propidium iodide kit (BD Biosciences, USA) and detected using flow cytometry using a FACS Calibur flow cytometer (Becton, Dickinson and Company, USA). MCF-7 cells were plated at a density of $2 \times 10^{5}$ cells/well into 12-well plates and incubated for 12 hours. The cells were then treated with different concentrations $(0,30,90$ and 120 $\mu \mathrm{M})$ of bergaptol for 48 hours. Cells grown in media containing an equivalent amount of $0.11 \%$ DMSO without any drug served as control. Cells were then collected and resuspended in binding buffer, incubated with annexin V-fluorescein isothiocyanate and propidium iodide for $30 \mathrm{~min}$ in the dark, prior to flow cytometric analysis.

\section{Flow cytometric evaluation of cell cycle arrest}

Human breast cancer cells (MCF-7) $\left(2 \times 10^{5}\right)$ were seeded in $60-\mathrm{mm}$ dishes and treated with different concentrations $(0,30,90$ and $120 \mu \mathrm{M})$ of bergaptol for 48 hours. Floating and adherent cells were trypsinized and washed twice with phosphate-buffered saline, then incubated in $70 \%$ ethanol at $-20^{\circ} \mathrm{C}$ overnight, treated with $20 \mu \mathrm{g} / \mathrm{mL}$ RNase A, then stained with $5 \mu \mathrm{g} / \mathrm{mL}$ of propidium iodide. Finally the stained cells were examined and studied by flow cytometry at wavelength of $488 \mathrm{~nm}$ [FACS Calibur instrument (BD Biosciences, USA) equipped with Cell Quest 3.3 software].

\section{Western blot analysis}

Western blot assay was done as previously reported (Ma et al., 2011) with slight modifications. Bradford assay (Bio-Rad) was used to determine the protein content. After electrophoresing a total of 20-40 Ag of protein on $15 \%$ SDS-PAGE gels, it was transferred to nitrocellulose membranes. Membranes were blocked, incubated with primary Abs at the suitable dose, and consequently incubated with primary antibody, washed and incubated with horseradish peroxidase-conjugated secondary antibody (1:2500 dilution; Bio-Rad). Detec- 
tion was performed using a chemiluminescent western detection kit (Cell Signaling Technology, Inc., USA).

\section{Statistical analysis}

The experiments were performed in triplicate. Data were expressed as the mean \pm standard deviation (SD). $\mathrm{p}<0.05$ was considered to indicate a statistically significant difference.

\section{Results}

\section{Effects of bergaptol on cell proliferation}

The results revealed that bergaptol exerted potent antiproliferative effects on MCF-7 cells (Figure 2). It showed concentration-dependent growth inhibitory effects against these cells. For determining the effectiveness of this compound, its $\mathrm{IC}_{50}$ value was also calculated to be $52.2 \mu \mathrm{M}$. Taxol which is a well-known anti-cancer drug was used as a positive control at a dose of $3.0 \mu \mathrm{M}$. It was observed that $120 \mu \mathrm{M}$ dose of bergaptol exhibited more potent growth inhibition of cancer cells than $3.0 \mu \mathrm{M}$ dose of taxol.

\section{Apoptosis induction as revealed by fluorescence microscopy using DAPI}

We next examined the apoptosis-related morphological effects caused by bergaptol in MCF-7 cancer cells using diamidine phenylindole dihrdrochloride (DAPI) staining involving fluorescence microscopy. Chromatin

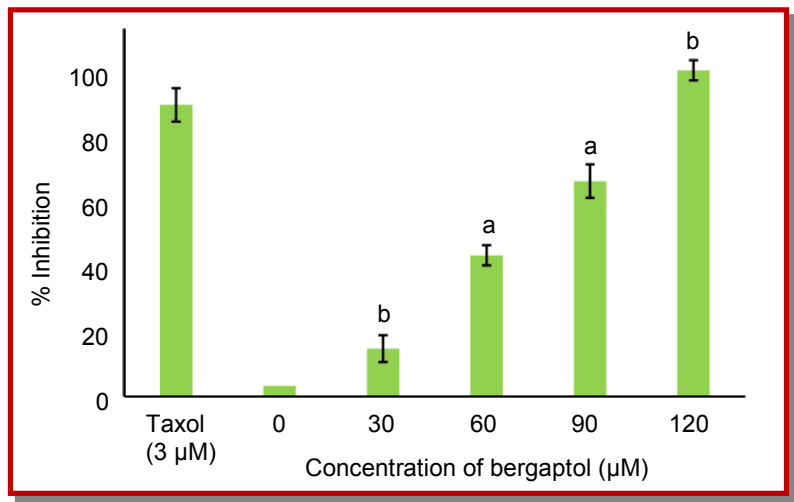

Figure 2: Cytotoxic effect of bergaptol in human breast cancer cells (MCF-7). Data are shown as the mean \pm SD of three independent experiments. ap $<0.05$; b $p<0.01$, vs $0 \mu \mathrm{M}$ (control)

condensation and chromosomal DNA cleavage were observed when MCF-7 cells were treated with bergaptol at different concentrations. As shown in Figure 3, with increase in concentration of bergaptol, there was increase in nuclear condensation and formation of apoptotic vesicles. Overall these results suggest that bergaptol was capable to induce apoptotic morphology in MCF-7 cells.

\section{Annexin V-FITC/PI assay for apoptosis evaluation and quantification}

Annexin V/propidium iodide double staining was used to detect apoptosis in the MCF-7 (Figure 4A-D). MCF-7

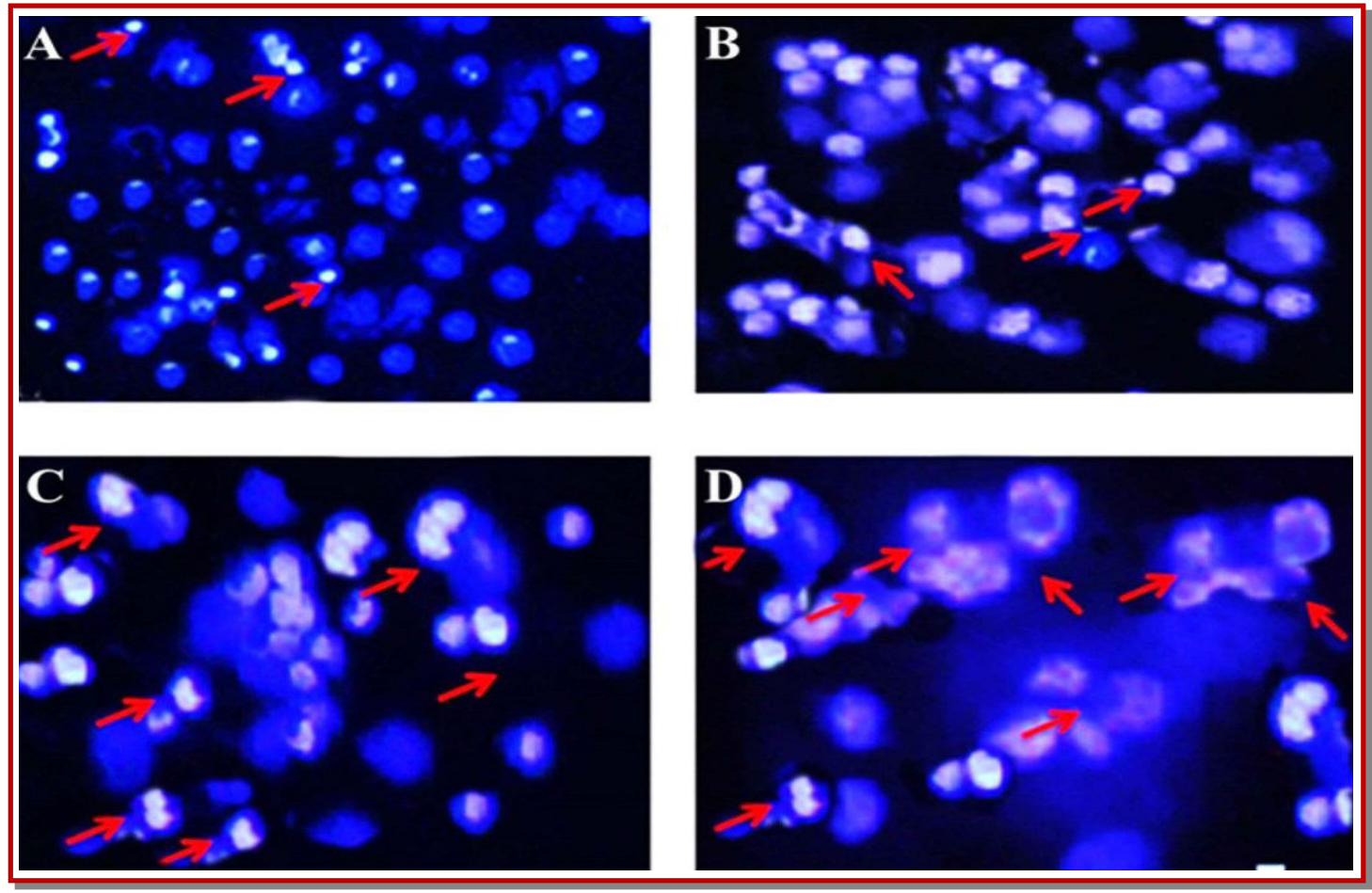

Figure 3: Fluorescence microscopy after MCF-7 cells were treated with different concentration (30, 90 and $120 \mu \mathrm{M})$ of bergaptol for 48 hours and stained with DAPI and analysed for nuclear condensation. Data are representative of one of the three independent experiments. A, represents the taxol-treated cells, while as B, C and D represent 30, 90 and $120 \mu \mathrm{M}$ of bergaptol respectively 
cells were treated with different concentration $(0,30,90$ and $120 \mu \mathrm{M}$ ) of bergaptol for 48 hours. Bergaptol induced both early and late apoptosis in a concentration -dependent manner (Figure 4B-D) as compared to the untreated control cells (Figure 4A). The different quadrants Q1, Q2, Q3 and Q4 represent necrotic cells, late apoptotic cells, viable cells and early apoptotic cell population respectively. Percentage of apoptotic cells increases from $8.5 \%$ in control cells (A), to $19.8,53.1$ and $87.2 \%$ in $25 \mu \mathrm{M}(\mathrm{B}), 75 \mu \mathrm{M}(\mathrm{C})$ and $125 \mu \mathrm{M}$ (D) bergaptol -treated cells respectively.

\section{Induction of cell cycle arrest}

With the aim of demonstrating whether bergaptol induces cell cycle arrest in MCF-7 cells, flow cytometric analysis using propidium iodide as a staining agent was performed after bergaptol treatment at different concentrations $(0,30,90$, and $120 \mu \mathrm{M})$ for 48 hours. As can be seen in Figure 5, after treatment with bergaptol, an increase in the proportion of cells in the S-phase (37.2, 45.3 and $65.1 \%$ as compared to $28.6 \%$ in untreated cells) and a reduction in the fraction of cells in the G1 phase $(44.1,41.6$ and $35.2 \%$ as compared to $51.2 \%$ in the untreated cells) was observed. Thus, bergaptol induces concentration dependent S-phase cell cycle arrest in human breast cancer cells.
Bergaptol induces cell apoptosis through mitochondrial pathway and caspase activation

To examine whether bergaptol induces apoptosis through this mechanism in MCF-7 cells, we evaluated the expression of cytochrome $c$, the pro-apoptotic protein Bax, the anti-apoptotic protein Bcl-2, caspase-3, caspase-9 and PARP by Western blot analysis. Bergaptol increased in a dose-dependent manner the cytosolic cytochrome c, Bax, cleaved caspase-3 and cleaved caspase- 9 expressions with a concomitant decrease in Bcl-2 expression compared to the untreated control (Figure 6). In the meantime, exposure of MCF-7 cells to bergaptol also led to cleavage of PARP fragment. The cleavage of PARP is considered to be a hallmark of cell apoptosis.

\section{Discussion}

The results revealed that bergaptol exerted potent antiproliferative effects on MCF-7 cancer cells. It showed concentration dependent growth inhibitory effects against these cells. Further, fluorescence microscopy revealed that chromatin condensation and chromosomal DNA cleavage were observed when MCF -7 cells were treated with bergaptol at different concen-

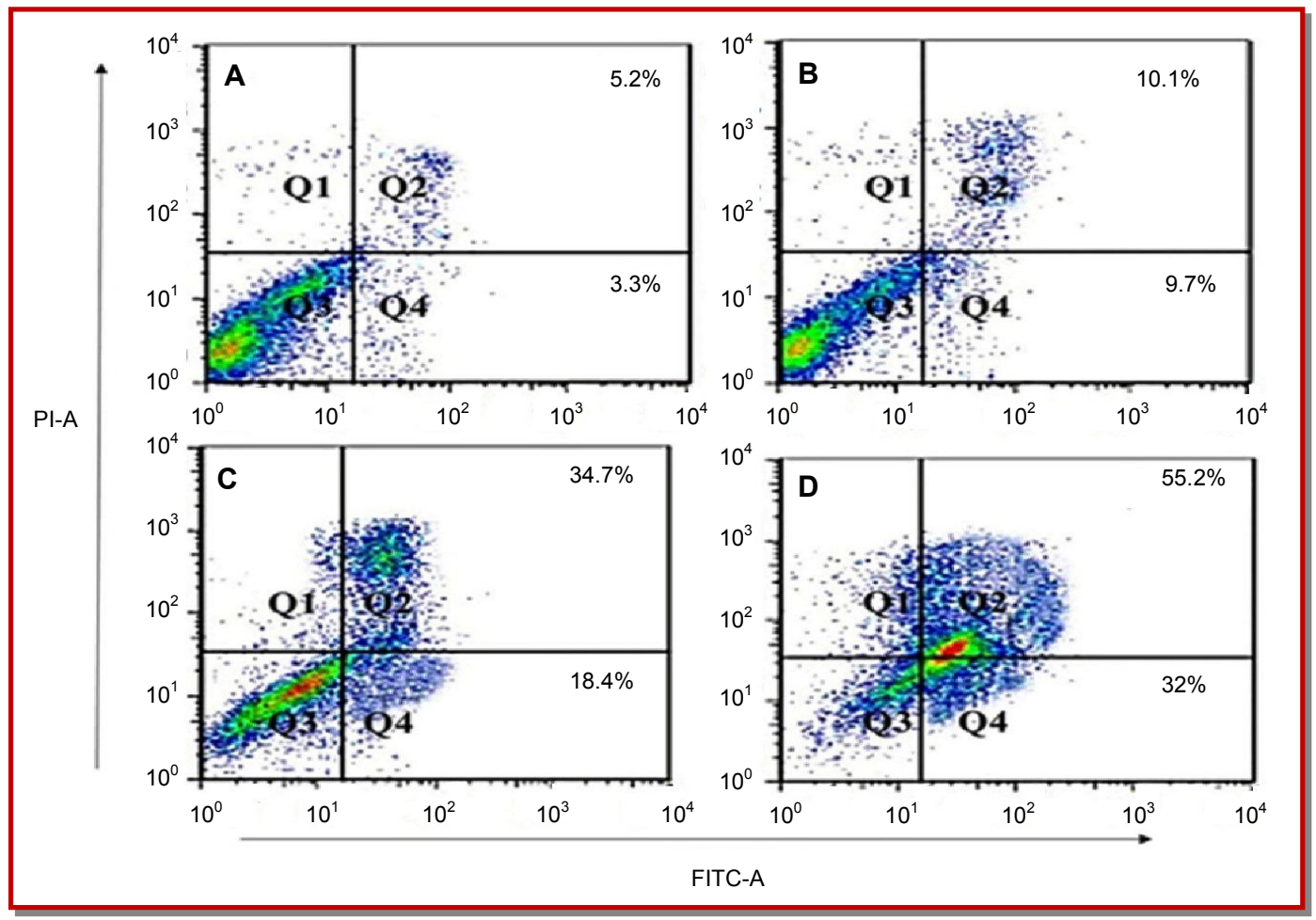

Figure 4: Quantification of bergaptol-induced apoptosis in human breast cancer cells (MCF-7). The cells were subjected to different doses of bergaptol $(0,30,90$ and $120 \mu \mathrm{M})$ for 48 hours and analyzed by flow cytometry with annexin V-FITC/propidium iodide staining. The different quadrants Q1, Q2, Q3 and Q4 represent necrotic cells, late apoptotic cells, viable cells and early apoptotic cell population respectively. Percentage of apoptotic cells increases from $8.5 \%$ in control cells (A), to $19.8 \%, 53.1 \%$ and $87.2 \%$ in $30 \mu \mathrm{M}(\mathrm{B}), 90 \mu \mathrm{M}(\mathrm{C})$ and $120 \mu \mathrm{M}$ (D) bergaptol-treated cells respectively 


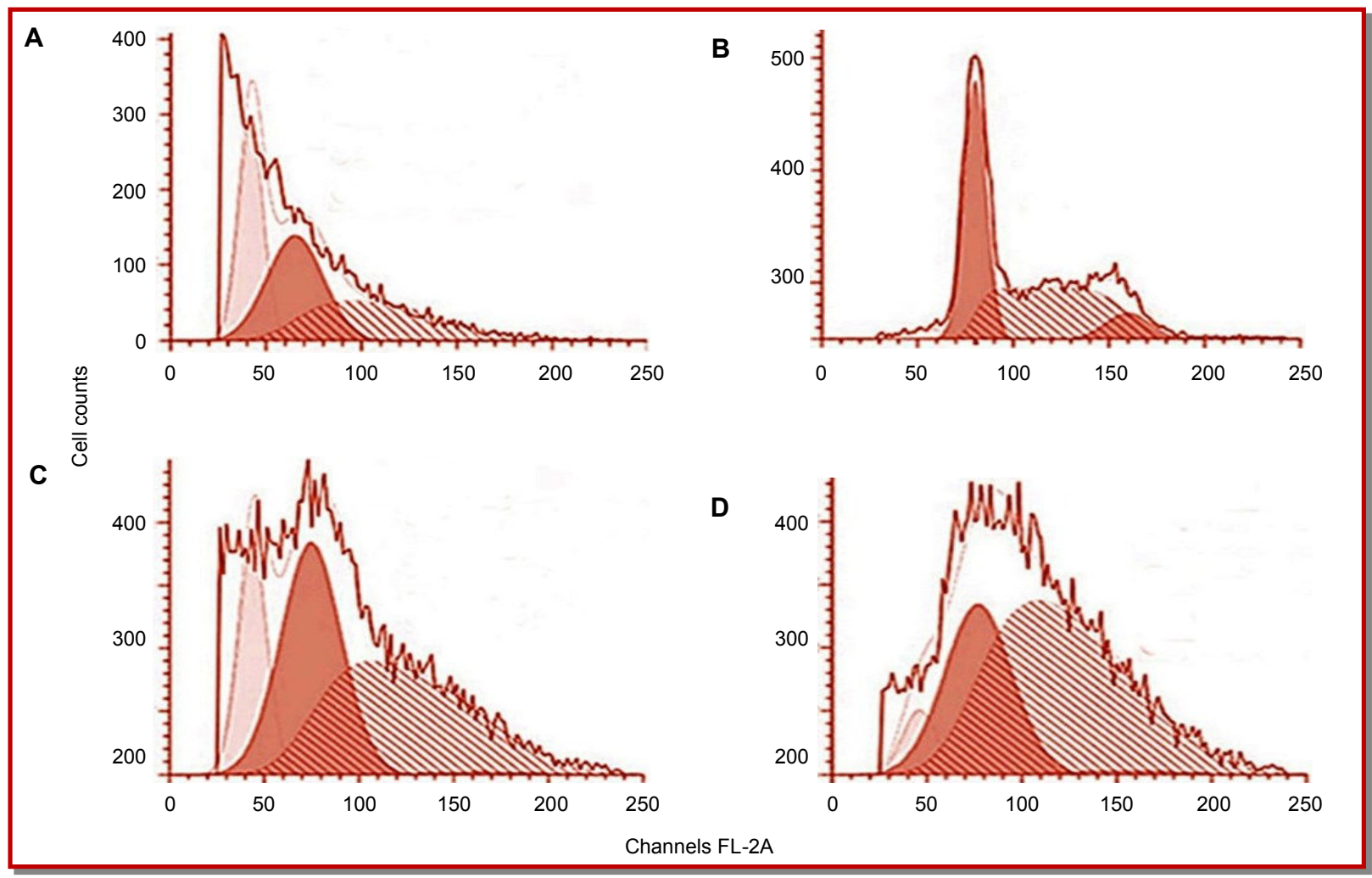

Figure 5: Bergaptol induces S-phase cell cycle arrest in in MCF-7 human breast cancer cells. Different doses of the compound were used, A represents untreated control, B represents $30 \mu \mathrm{M}, \mathrm{C}$ represents $90 \mu \mathrm{M}$ and D represents $120 \mu \mathrm{M}$ dose of bergaptol respectively. After treatment, cells were harvested and stained with propidium iodide, and then analyzed by flow cytometry

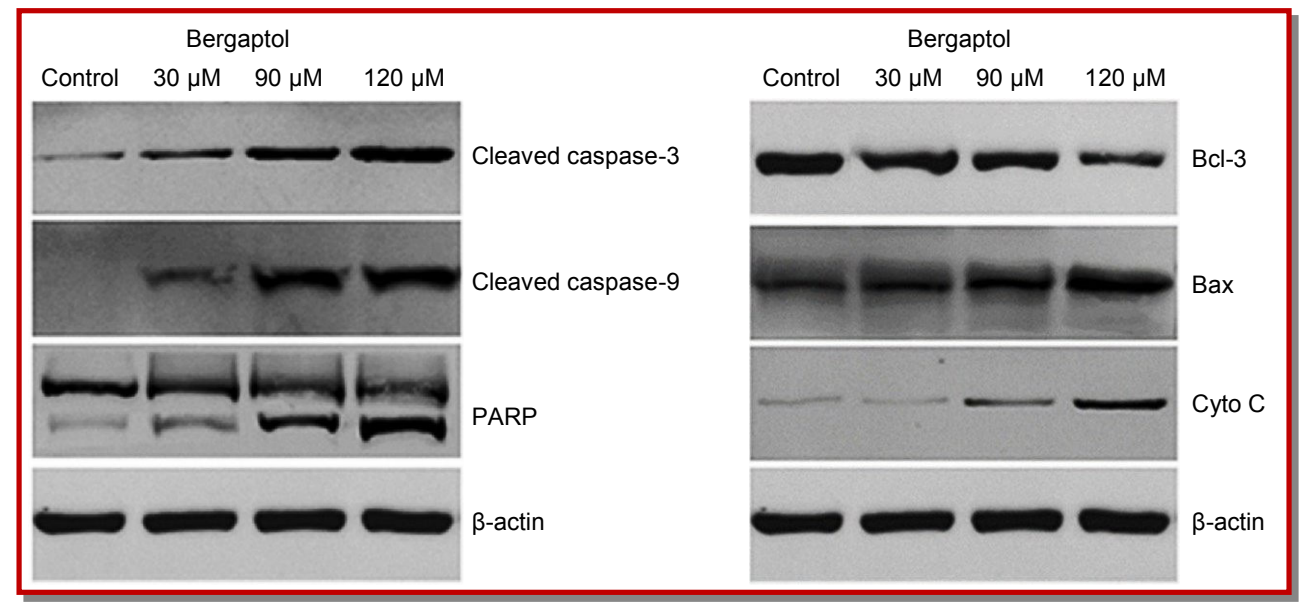

Figure 6: The effect of bergaptol on the expression of caspase-dependent mitochondrial apoptosis pathway proteins in MCF-7 breast cancer cells. Representative images of cytochrome c, Bax, Bcl-2, PARP, cleaved caspase-9 and cleaved caspase-3 protein expression detected by Western blot. $\beta$-actin was used as a control

trations. Annexin V-FITC/propidium iodide assay for apoptosis quantification showed that bergaptol induced both early and late apoptosis. Percentage of apoptotic cells increases from $8.5 \%$ in control cells, to $19.8,53.1$ and $87.2 \%$ in $25 \mu \mathrm{M}, 75 \mu \mathrm{M}$ and $125 \mu \mathrm{M}$ bergaptoltreated cells respectively. Cell cycle analysis by flow cytometry revealed that bergaptol induced concentration dependent S-phase cell cycle arrest in human breast cancer cells. Western blotting revealed that bergaptol increased the cytosolic cytochrome c, Bax, cleaved caspase- 3 and cleaved caspase- 9 expressions with a concomitant decrease in Bcl-2 expression compared to the untreated control.

Suppression of cancer growth often involves modulation of various key signaling pathways which results in altered gene expression, disturbances in cell cycle or apoptotic process. Apoptosis is a highly organized cell 
suicide process which leads to the removal of damaged cells. There are various morphological features that characterize the process of apoptosis including cell shrinkage and rounding because of the collapse of the cytoskeleton by caspases. This is followed by chromatin condensation and DNA fragmentation (Elmore, 2007; Böhm, 2003; Susin et al., 2000). Apoptosis process has been recently shown to suggest promising therapeutic targets for the design and development of novel anticancer agents (Khoo et al., 2010). Natural product-based bioactive compounds have always played key roles in the anti-cancer drug discovery program. Natural products have been found to act as chemo preventive agents with the tendency to induce apoptosis in several in vitro and in vivo models of carcinogenesis (Molinari, 2009; Koehn and Carter, 2005). Many conventional anticancer drugs are presently used in anti-cancer chemotherapy which are suggested to induce cell apoptosis through the stimulation of these pathways (Ponci et al., 2015). As a result, this tendency of natural products to induce apoptotic cell death has become a major mechanism for the treatment of cancer.

\section{Conclusion}

Bergaptol exhibits anti-cancer activity in MCF-7 breast carcinoma cells by inducing mitochondrial mediated apoptosis and cell cycle arrest.

\section{Conflict of Interest}

The authors declare that there is no conflict of interest to reveal.

\section{References}

Böhm I. Disruption of the cytoskeleton after apoptosis induction by autoantibodies. Autoimmunity 2003; 36: 183-89.
Conforti F, Menichini F. Phenolic compounds from plants as nitric oxide production inhibitors. Curr Med Chem. 2011; 18: 1137-45.

Eccles SA, Aboagye EO, Ali S, Anderson AS, Armes J, Berditchevski F, Blaydes JP, Brennan K, Brown NJ, Bryant $\mathrm{HE}$, Critical research gaps and translational priorities for the successful prevention and treatment of breast cancer, Breast Cancer Res. 2013; 15: R92.

Elmore S. Apoptosis: A review of programmed cell death. Toxicol Pathol. 2007; 35: 495-516.

Guzman-Villanueva D, El-Sherbiny IM, Herrera-Ruiz D, Smyth HD. Design and in vitro evaluation of a new nanomicroparticulate system for enhanced aqueous-phase solubility of curcumin. Biomed Res Int. 2013; 2013: 724763.

Jemal, A, Bray F, Center MM, Felay J, Ward E, Forman D. Global cancer statistics. CA-Cancer J Clin. 2011; 61: 69-90.

Khoo BY, Chua SL, Balaram P. Apoptotic effects of chrysin in human cancer cell lines. Int J Mol Sci. 2010; 11: 2188-99.

Koehn FE, Carter GT. The evolving role of natural products in drug discovery. Nat Rev Drug Discov. 2005; 4: 206-20.

Ma L, Wen ZS, Liu Z, Hu Z, Ma J, Chen XQ, Liu YQ, Pu JX, Xiao WL, Sun HD, Zhou GB. Overexpression and small molecule-triggered down-regulation of CIP2A in lung cancer. PLoS One. 2011; 6: e20159.

Molinari G. Natural products in drug discovery: Present status and perspectives. Adv Exp Med Biol. 2009; 655: 13-27.

Newman DJ, Cragg GM. Natural products as sources of new drugs over the last 25 years. J Nat Prod. 2007; 70: 461-77.

Ponci V, Figueiredo CR, Massaoka MH, de Farias CF, Matsuo $\mathrm{AL}$, Sartorelli P, Lago JH. Neolignans from Nectandra megapotamica (Lauraceae) display in vitro cytotoxic activity and induce apoptosis in leukemia cells. Molecules 2015; 20: 2757-68.

Stanley WL, Vannier SH. Chemical composition of lemon oil. I. Isolation of a series of substituted coumarins. J Am Chem Soc. 1957; 79: 3488-91.

Susin SA, Daugas E, Ravagnan L, Samejima K, Zamzami N, Loeffler M, Costantini P, Ferri KF, Irinopoulou T, Prévost MC, Brothers G, Mak TW, Penninger J, Earnshaw WC, Kroemer G. Two distinct pathways leading to nuclear apoptosis. J Exp Med. 2000; 192: 571-80. 


\section{Your feedback about this paper}

1. Number of times you have read this paper 0

2. Quality of paper
Excellent
Good
Moderate
Not good

3. Your comments

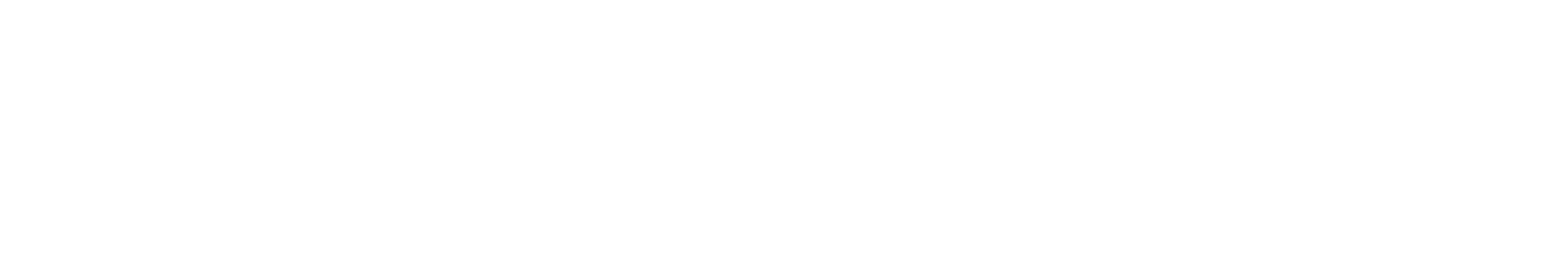

